\title{
Amplitude-masked photoacoustic wavefront shaping: theory and application in flowmetry
}

Jinyang Liang, Jian Wei Tay, Ashton S. Hemphill, Lihong V. Wang

Jinyang Liang, Jian Wei Tay, Ashton S. Hemphill, Lihong V. Wang, "Amplitude-masked photoacoustic wavefront shaping: theory and application in flowmetry," Proc. SPIE 9323, Photons Plus Ultrasound: Imaging and Sensing 2015, 932310 (11 March 2015); doi: 10.1117/12.2081693

SPIE. Event: SPIE BiOS, 2015, San Francisco, California, United States 


\title{
Amplitude-masked Photoacoustic Wavefront Shaping: Theory and Application in Flowmetry \\ Jinyang Liang $\dagger$, Jian Wei Taył, Ashton S. Hemphill, and Lihong V. Wang* \\ Optical Imaging Laboratory, Department of Biomedical Engineering, Washington University in St \\ Louis, One Brookings Drive, St Louis, MO 63130 \\ $\dagger$ These authors contribute equally to this work.
}

\begin{abstract}
Optical diffusion in scattering media prevents focusing beyond shallow depths, causing optical imaging and sensing to suffer from low optical intensities, resulting in low signal-to-noise ratios (SNR). Here, we demonstrate focusing using a fast binary-amplitude digital micromirror device to characterize the transmission modes of the scattering medium. We then identify and selectively illuminate the transmission modes which contribute constructively to the intensity at the optical focus. Applying this method to photoacoustic flowmetry, we increased the optical intensity at the focus six-fold, and showed that the corresponding increase in SNR allows particle flow to be measured.
\end{abstract}

Keywords: Active or adaptive optics, Photoacoustics, Turbid media

\section{INTRODUCTION}

Light propagating in optically scattering media, such as biological tissue, becomes distorted, limiting focusing in such media to depths less than one transport mean free path (approximately $1 \mathrm{~mm}$ in soft tissue [1]). This limitation affects both the operational depth and resolution of optical techniques, such as imaging and phototherapy. Recently, optical wavefront shaping [2-10] has made considerable progress in focusing light into scattering media. The process of light scattering and propagation can be expressed by a transmission matrix [9]. Each element in the transmission matrix, $t_{m n}$, links the $m$-th mode of the output optical field with the $n$-th mode in the input optical field. For a typical scattering medium, light passing through all elements of the transmission matrix contributes to the output optical field.

A common approach in wavefront shaping is to measure the transmission matrix and modulate the phase or/and amplitude of the incident laser beam to compensate for scattering and focus light within the medium. Liquid crystal spatial light modulators (LCSLMs) are commonly used to shape the phase profile of the illuminating wavefront. The optimal phase pattern can be determined by using either iterative optimization algorithms [3,6-8] or by directly measuring and inverting the transmission matrix [9]. In general, these algorithms display different phase patterns on the SLM, and they measure the change in the optical field either directly (e.g., by using a CCD camera [8,9]) or indirectly (e.g., by using photoacoustics [5,7,10] or ultrasonic modulation [6]). Of these methods, photoacoustic-guided wavefront shaping is particularly promising as a non-invasive technique. Photoacoustic (PA) signals are generated when an absorbing target is illuminated by a laser pulse. For biological applications, PA detection of endogenous absorption, for example from blood, provides a label-free contrast mechanism [11].

For optical wavefront shaping to work, the optimized phase pattern must be obtained and displayed on the SLM within the speckle decorrelation time of the object, which is the length of time that the speckle remains stationary, preserving most scattering path-length variations within one optical wavelength. For live tissue, this decorrelation time is on the order of 1 millisecond, depending on the probing depth. However, the frame rates of LC-SLMs are only $100 \mathrm{fps}$, much too slow. Alternatively, digital micromirror devices (DMDs), which have switching speeds up to tens of kilohertz, can be used. These devices are binary-amplitude SLMs; each element is a micromirror which can be toggled between on or off states. Digital holograms (Lee holograms) can be used to generate the phase patterns; however, due to low diffraction efficiency, only $\sim 20 \%$ of the incident light is usable [4]. For a given laser intensity, the low modulation efficiency could transfer to a low signal-to-noise ratio (SNR) in the measurements, making DMDs impractical as phasemodulators.

*Corresponding author: LHWANG@WUSTL.EDU

Photons Plus Ultrasound: Imaging and Sensing 2015, edited by Alexander A. Oraevsky, Lihong V. Wang

Proc. of SPIE Vol. 9323, 932310 - @ 2015 SPIE · CCC code: 1605-7422/15/\$18

doi: $10.1117 / 12.2081693$

Proc. of SPIE Vol. $9323932310-1$ 
Alternatively, amplitude-masked wavefront shaping can be used [12] [Fig 1 (a)]. In this technique, the optical modes which do not contribute constructively to the optical focus are rejected by turning off the corresponding DMD segments. The remaining modes therefore add to form an optical focus [Fig 1 (b)]. Previously, the optimized DMD pattern was obtained by using the continuous sequential (CS) algorithm, where the change in the signal was measured by changing a single segment at a time [12]. However, in this case, the signal arises from just a single DMD segment, resulting in a low SNR [13], which contributes to measurement errors and results in lower focusing efficiencies. To address these issues, we propose amplitude-masked photoacoustic wavefront shaping (AMPAWS). Our implementation utilizes a DMD to increase measurement speed, and Hadamard multiplexing to increase measurement SNR. We demonstrate the usefulness of AM-PAWS by applying it to increase the SNR of photoacoustic flowmetry.

(a)

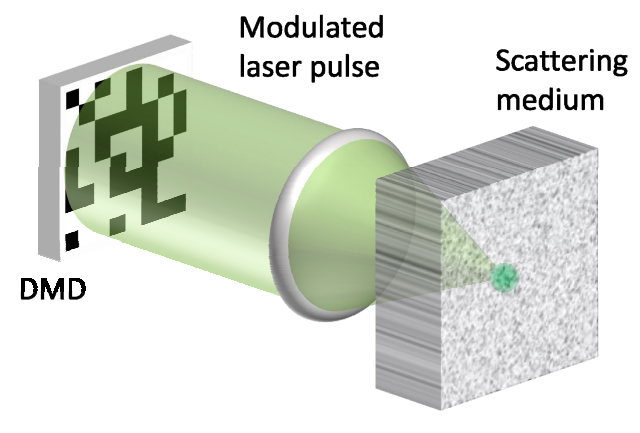

(b)

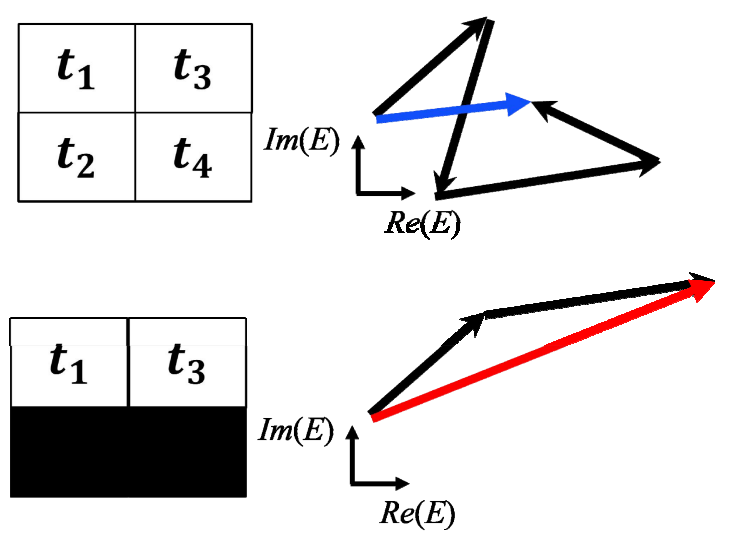

Fig. 1 (a) Principle of amplitude-masked wavefront shaping based on a DMD. (b) Vector analysis of output optical field with a fourelement transmission matrix $\left(t_{n}, n=1,2,3,4\right)$. Top: output optical field with a non-modulated laser pulse. Note that destructive interference reduces the amplitude of the output optical field. Bottom: output optical field with an amplitude-masked laser pulse. Note that the amplitude mask turns off elements interfering destructively and increases the amplitude of the resultant output optical field.

\section{THEORY}

Herein, we explain how our measurement procedure works in mathematical detail [14]. An optical field mode $E^{\text {out }}$ beyond a scattering medium is related to the input optical field by

$$
E^{\text {out }}=\sum_{n=1}^{N} t_{n} E_{n}^{i n}=\sum_{n=1}^{N} A_{n} e^{i \Phi_{n}},
$$

where $t_{n}$ is an element of the transmission matrix, with amplitude $A_{n}$ and phase $\Phi_{n}$, and $E_{n}^{i n}$ is the optical field of the $n$-th input mode, where each mode represents the field contribution from each independently controlled DMD segment. $N$ is therefore the total number of these segments. We have also assumed that $\arg \left(E_{n}^{i n}\right)=0$ and $\left|E_{n}^{i n}\right|=1$. To perform the multiplexing, we used the concept of "virtual elements" formed by linear combinations of the DMD micromirrors using the Hadamard basis, as introduced by Herbert et al. for ultrasound transducers [13]. The electric field $\varepsilon_{m}$ generated by the $m$-th combination is then given by

$$
\varepsilon_{m}=\sum_{n=1}^{N} H_{m n} A_{n} e^{i \Phi_{n}}=B_{m} e^{i \Psi_{m}},
$$

where the elements $H_{m n}$ are taken row-wise from a Hadamard matrix of order $N$, and $B_{m}$ and $\Psi_{m}$ are the amplitude and phase of the resultant optical field. Each element $H_{m n}$ is either $-1 / 2$ or $+1 / 2$.

To measure the transmission, we displayed binary patterns on the DMD. These patterns consist of 0's or 1's, corresponding to micromirrors turned off or on. Each DMD pattern is therefore equal to the combination of the first and subsequent virtual elements, i.e., the resulting electric field is equal to $\varepsilon_{1}+\varepsilon_{m}$, which automatically defines $\varepsilon_{1}$ as the reference vector. Here, $\varepsilon_{1}$ is simply the 
resultant vector when all the DMD pixels are turned on, i.e., $H_{1 n}=1 / 2$. The measured intensity when the $m$-th pattern is displayed is then given by

$$
I_{m}=\left(\varepsilon_{1}+\varepsilon_{m}\right)\left(\varepsilon_{1}+\varepsilon_{m}\right)^{*}=\left|\varepsilon_{1}\right|^{2}+\left|\varepsilon_{m}\right|^{2}+2 \operatorname{Re}\left\{B_{m} e^{i \Psi_{m}}\right\},
$$

where we have set $\left|B_{1}\right|=1$ and $\Psi_{1}=0$ without loss of generality. Note that the first two terms are constant, and consequently the measurements can be differentiated by the sign of the third term. This sign depends on the phase angle $\Psi_{m}$, which is measured with respect to the vector $\varepsilon_{1}$. Noting that $\operatorname{Re}\left\{\varepsilon_{m}\right\}=\Sigma_{n=1}^{N} H_{m n} \operatorname{Re}\left\{A_{n} e^{i \Phi_{n}}\right\}=\operatorname{Re}\left\{B_{m} e^{i \Psi_{m}}\right\}$, the optimized pattern can be obtained by multiplying the measured values of $I_{m}$ by the inverse of the Hadamard matrix, then turning on only the segments that have positive values. This is equivalent to selecting the segments that have phase $\Phi_{m} \in[-\pi / 2, \pi / 2]$, measured relative to $\Psi_{1}$, and that therefore interfere constructively at the focus.

So far, we have assumed that a single output mode is measured directly, e.g., by using a photodiode. In our experiments though, $I_{m}$ is measured indirectly using an ultrasonic transducer to detect PA signals generated from the target. Nevertheless, since the PA signals are linearly related to the total energy absorbed [10], this procedure is still valid. In this case, the peak-to-peak PA signal amplitude is proportional to the total optical fluence $F_{m}\left(\mathrm{~J} / \mathrm{m}^{2}\right)$ absorbed within the ultrasound detection volume $V$ :

$$
p_{m} \propto \int \mu_{a}(x, y, z) F_{m}(x, y, z) d V,
$$

where $F_{m}=\int I_{m}(t) d t$ is integrated over the laser pulse time, $\mathrm{x}$ and $\mathrm{y}$ are along the transducer's transverse plane, $\mathrm{z}$ is along the light propagation direction, and $\mu_{a}$ is the absorption coefficient.

\section{SYSTEM}

The optical setup of our system [14] is shown schematically in Fig. 2. We used a $523 \mathrm{~nm}$ pulsed laser beam (Nd:YLF, EdgeWave, BX-series), with a pulse energy of $\sim 800 \mu \mathrm{J}$ and a repetition rate of $1 \mathrm{kHz}$. The beam was expanded to $\sim 2 \mathrm{~cm}$ in diameter to fill the aperture of the DMD (Texas Instruments, D4100; 1024×768 pixels), giving a pulse energy density of $\sim 200 \mu \mathrm{J} / \mathrm{cm}^{2}$ at the DMD surface. We note that this was the strongest pulse energy that could be handled by the DMD before we started to observe malfunctioning pixels. The beam was then reduced to $5 \mathrm{~mm}$ and focused on to the sample by a $10 \times$ objective lens (N.A. $=0.25$ ). A small portion of the beam was also directed to a photodiode and used to compensate for pulse-to-pulse energy fluctuations.

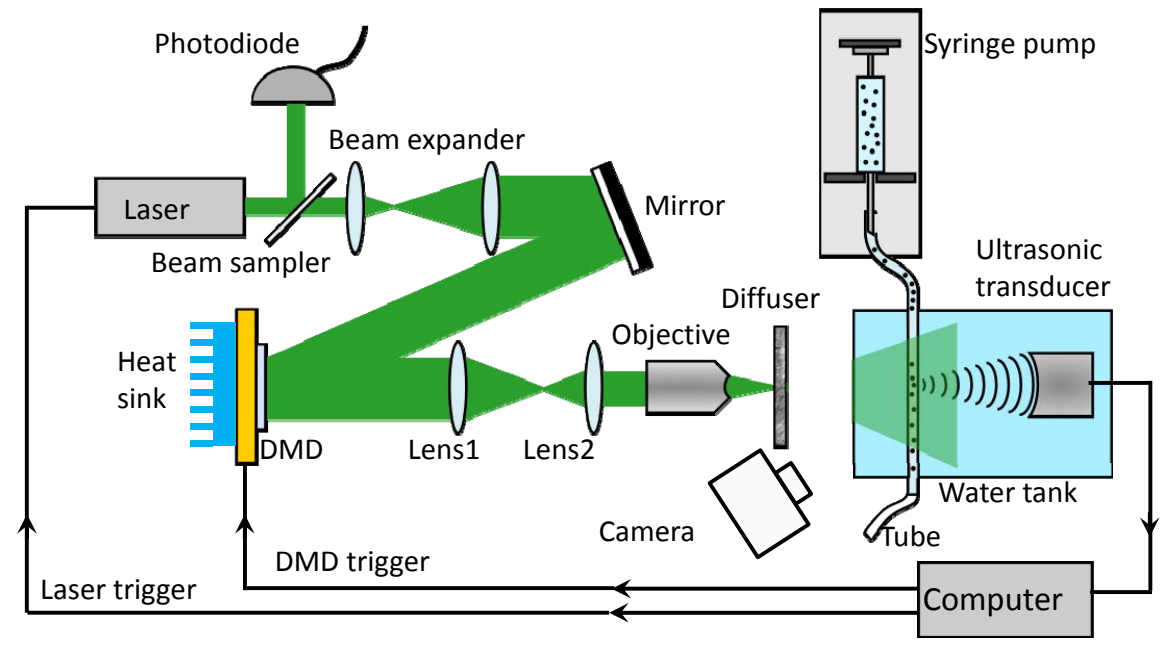

Fig. 2. Schematic representation of the optical system. DMD: digital micromirror device. 
To demonstrate the usefulness of our method, we applied it to photoacoustic flowmetry. Specifically, we placed a silicone tube (Silastic, $1 \mathrm{~mm}$ inner diameter) $\sim 14 \mathrm{~cm}$ behind a ground-glass diffuser (Thorlabs, DG10-120) as a mimic a blood vessel within tissue. The tube was submerged in water for acoustic coupling. The PA signal was detected using a $10 \mathrm{MHz}$ ultrasonic transducer (Panametrics, A315S; $\mathrm{f} \#=2,-6 \mathrm{~dB}$ bandwidth $=5.5 \mathrm{MHz}$ ), which had a $400 \mu \mathrm{m}$ transverse focal diameter, measured as the FWHM of the transducer response profile. The speckle size after the diffuser was $440-660 \mu \mathrm{m}$, measured using the autocorrelation of a CCD image of the speckle field [15], giving a single speckle within the ultrasonic focus. In addition, a CCD camera (Point Grey, FMVU03MTM-CS) was added to capture the particle flow as the reference.

\section{EXPERIMENT}

\subsection{Optimization of the DMD pattern}

The experiment was carried out in two stages: optimization as described previously, and measurement of particle flow. During the optimization stage, we filled the tube with black ink to mimic homogeneous absorption in flowing blood. We divided the DMD into $1024(32 \times 32)$ independent segments, with each segment consisting of $32 \times 24$ micromirrors. The 1024 patterns required were generated from a Hadamard matrix of the corresponding order. Each pattern was displayed on the DMD, and the resulting PA signals were amplified 100× (Minicircuits, ZFL-500LN), and measured with an oscilloscope (Tektronix, DPO2024). Initially, no PA signal was detected using single-shot measurements, and averaging was required. The PA signal was averaged over 64 acquisitions, increasing the SNR to 3.9. The SNR was measured by calculating the ratio of the PA signal amplitude and the standard deviation of the noise - acquired when no light was present. Then the measured PA signal amplitudes were inverse Hadamard transformed to calculate the optimal pattern. We compared the PA signal from the optimal pattern with the PA signal generated by turning all the segments on (uniform), and from a randomized pattern with the same number of segments turned on. Note that the randomized pattern generates a PA signal that is $\sim 2 \times$ smaller than the signal when all the DMD pixels are turned on. The optimized PA signal amplitude was $14 \times$ larger than that of the randomized pattern, and $6.5 \times$ larger than that of the uniform pattern. At first glance, this result may appear counter-intuitive; an increase in optical intensity was obtained despite approximately half the micromirrors being turned off in the optimal pattern, resulting in half as much total incident light delivered on the sample. Nevertheless, the intensity was increased because we selected the segments which added constructively at the transducer focus.

The expected intensity increase compared to the randomized pattern can be estimated by [12]

$$
\eta \approx 1+\frac{1}{\pi}\left(\frac{N}{2}-1\right) .
$$

$N=1024$ in our experiment, and hence, the expected increase was $\sim 163$, assuming that only a single speckle was measured. The intensity increase is expected to be reduced proportionally by the number of modes within the detection volume [5]. The lower actual improvement obtained was most likely due to the poor SNR in the measurement stage, as well as uneven DMD illumination (the optical intensity varied by as much as $2 \times$ due to the high order harmonics in the incident laser beam).

\subsection{Measurements of particle flow}

After optimization, the single-shot SNR was increased to $\sim 8$, easily sufficient to enable flow measurements. Thus, in the second stage, we replaced the black ink with an aqueous suspension of microspheres (Phosphorex, 1500KR, red polystyrene), which had an average diameter of $500 \mu \mathrm{m}$ (variation $\sim 10 \%$ ), chosen to match the detection volume. The microspheres were strongly absorbing and generated broadband PA signals. The flow speed of the suspension through the tube was controlled by a syringe pump (Braintree Scientific, BSP-99M) set between 0.3 and $1.8 \mathrm{~mm} / \mathrm{s}$, which is comparable to capillary blood flows [16]. The speed of the particles in the suspension was measured using PA correlation [17]: first, a series of PA signals (A-lines), detected by the ultrasonic transducer as the particles traversed the generated optical focal spot, were amplified $100 \times$ as before, then measured using a 12-bit digitizer card (AlazarTech, ATS9350) at a sampling rate of $500 \mathrm{MS} / \mathrm{s}$. Each A-line was recorded from the PA signal generated by a single laser pulse (single-shot). The peak-to-peak amplitude for each PA A-line in the series was then calculated and used as one data point of the "slow-time" profile $[17,18]$. This "slow-time" profile refers to the millisecond-scale measurement time at each laser pulse, as opposed to the "fast time", which is the microsecond-scale PA flight time in each A-line. For comparison, Figs. 3(a) and (b) show the measured slow-time PA profiles when the syringe pump was set to $0.58 \mathrm{~mm} / \mathrm{s}$, and with either the uniformly (all "on") or the optimal 
pattern displayed. As can be seen in Fig. 3(a), even with all the DMD micromirrors turned on, there was insufficient SNR for singleshot measurements. However, as shown in Fig. 3(b), after the optimization, the increased SNR allowed the particles to be clearly detected.

We then calculated the normalized autocorrelation function $G(\tau)$ of each slow-time profile, which is related to the flow speed by [17]

$$
G(\tau)=\exp \left(-\frac{\tau}{\tau_{f}}\right)^{2},
$$

where $\tau$ is the slow time, and $\tau_{f}=r_{0} / v_{f}$ is the decay constant, given by the ratio of the detection spot size $r_{0}$ and the particle flow speed $v_{f}$. The detection spot size $r_{0}=\sqrt{r_{p}^{2}+r_{U S}^{2}}$ is given by the convolution of the particle diameter $r_{p}$ and the generated optical spot size, which is assumed to be equal to the full-width at half maximum of the transducer detection sensitivity $r_{U S}$. To obtain $v_{f}$, the slow-time profile of each particle was fitted to Eq. (6). As shown in Fig. 3(c), the measured flow speeds and the preset speeds are in agreement. The variation in the measured speed is mainly due to variations in particle size. In addition, the larger particles tended to sink and drag along the bottom of the tube.
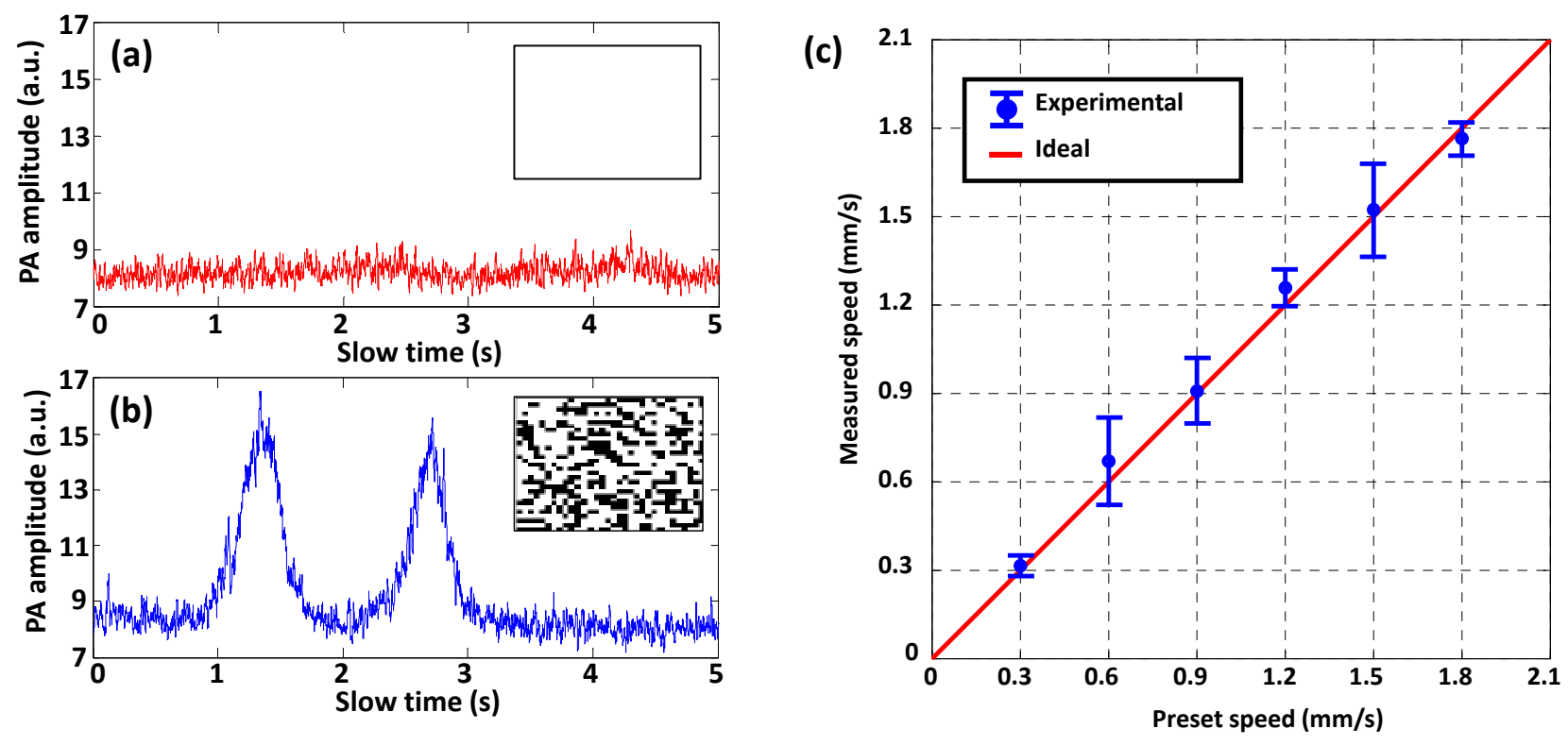

Fig. 3. Measurements of particle flow. Slow-time PA profiles for (a) a uniform pattern (all on) and (b) the optimized pattern displayed on the DMD. The insets show the DMD patterns. Note that the signal in (a) is similar to the noise level in (b). (c) Flow speed measured with different settings of the syringe pump, using the optimized DMD pattern. The error bars indicate the standard deviation.

\section{SUMMARY}

In conclusion, we have demonstrated amplitude-masked PAWS and proved its usefulness in photoacoustic flowmetry. By using binary amplitude masking, an increase in intensity of $14 \times$ over the initial diffuse illumination was obtained. This intensity increase gave a corresponding increase in measurement SNR, readily allowing single particle flow speeds to be measured. At the current stage, the limited ability of the DMD to handle strong laser pulses is the main challenge that needs to be overcome before our technique can be used with real tissue. During initial testing, we started to observe DMD malfunction when the pulse energy density exceeded 200 $\mu \mathrm{J} / \mathrm{cm}^{2}$. The limited energy handling ability causes two consequences. First, each PA amplitude is averaged by 64 acquisitions, and the entire optimization process can take up to $\sim 2$ minutes. Due to this insufficient speed, we used a ground glass diffuser as the static scattering medium. However, the speed is not a fundamental limitation, as the DMD is capable of operating at $22 \mathrm{kHz}$. Therefore, with sufficient SNR, the measurement time could have been reduced to $47 \mathrm{~ms}$, which is sufficient for some biological applications. 
Second, the relatively low SNR limited the measurement precision, which caused error in the optimized pattern. Therefore, the experimentally determined PA signal enhancement was lower than the theoretical prediction. Both consequences can be avoided if the DMD has a higher laser pulse energy handling ability. Finally, in this experiment, the speckles were relatively large. In deep tissue, speckle sizes are comparable to the laser wavelength, giving approximately 0.5 million speckles within the detection volume. State-ofthe-art DMDs currently have resolutions of $\sim 2$ million pixels and cannot provide adequate control. However, the number of detected (and therefore controlled) speckles could be reduced by using nonlinear PA signals [7] or by filtering the transducer response [19, 20].

\section{ACKNOWLEDGMENTS}

We would like to thank Yong Zhou for experimental assistance and Professor James Ballard for assistance in proofreading the manuscript. This work was sponsored in part by the National Institutes of Health grants DP1 EB016986 (NIH Director's Pioneer Award), R01 CA186567 (NIH Director's Transformative Research Award), R01CA157277, and R01 CA159959. L. V. Wang has a financial interest in Microphotoacoustics, Inc. and Endra, Inc., which, however, did not support this work.

\section{REFERENCES}

[1] Wang, L. V., and Wu, H.-i., [Biomedical optics: principles and imaging] John Wiley \& Sons, (2012).

[2] Vellekoop, I. M., and Mosk, A., "Focusing coherent light through opaque strongly scattering media," Opt. Lett., 32(16), 2309-2311 (2007).

[3] Conkey, D. B., Brown, A. N., Caravaca-Aguirre, A. M., and Piestun, R., "Genetic algorithm optimization for focusing through turbid media in noisy environments," Opt. Express, 20(5), 4840-4849 (2012).

[4] Conkey, D. B., Caravaca-Aguirre, A. M., and Piestun, R., "High-speed scattering medium characterization with application to focusing light through turbid media," Opt. Express, 20(2), 1733-1740 (2012).

[5] Chaigne, T., Katz, O., Boccara, A. C., Fink, M., Bossy, E., and Gigan, S., "Controlling light in scattering media non-invasively using the photoacoustic transmission matrix," Nature Photonics, 8(1), 58-64 (2014).

[6] Tay, J. W., Lai, P., Suzuki, Y., and Wang, L. V., "Ultrasonically encoded wavefront shaping for focusing into random media," Scientific reports, 4, (2014).

[7] Lai, P., Wang, L., Tay, J. W., and Wang, L. V., "Photoacoustically guided wavefront shaping for enhanced optical focusing in scattering media," Nature Photonics, (2015).

[8] Vellekoop, I., and Mosk, A., "Phase control algorithms for focusing light through turbid media," Optics Communications, 281(11), 3071-3080 (2008).

[9] Popoff, S., Lerosey, G., Carminati, R., Fink, M., Boccara, A., and Gigan, S., "Measuring the transmission matrix in optics: an approach to the study and control of light propagation in disordered media," Physical review letters, 104(10), 100601 (2010).

[10] Kong, F., Silverman, R. H., Liu, L., Chitnis, P. V., Lee, K. K., and Chen, Y.-C., "Photoacoustic-guided convergence of light through optically diffusive media," Opt. Lett., 36(11), 2053-2055 (2011).

[11] Wang, L. V., and Hu, S., "Photoacoustic Tomography: In Vivo Imaging from Organelles to Organs," Science, 335(6075), 1458-1462 (2012).

[12] Akbulut, D., Huisman, T. J., van Putten, E. G., Vos, W. L., and Mosk, A. P., "Focusing light through random photonic media by binary amplitude modulation," Opt. Express, 19(5), 4017-4029 (2011).

[13] Herbert, E., Pernot, M., Montaldo, G., Fink, M., and Tanter, M., "Energy-based adaptive focusing of waves: application to noninvasive aberration correction of ultrasonic wavefields," Ultrasonics, Ferroelectrics and Frequency Control, IEEE Transactions on, 56(11), 2388-2399 (2009).

[14] Tay, J. W., Liang, J., and Wang, L. V., "Amplitude-masked photoacoustic wavefront shaping and application in flowmetry," Opt. Lett., 39(19), 5499-5502 (2014).

[15] Piederrière, Y., Cariou, J., Guern, Y., Le Jeune, B., Le Brun, G., and Lortrian, J., "Scattering through fluids: speckle size measurement and Monte Carlo simulations close to and into the multiple scattering," Opt. Express, 12(1), 176-188 (2004).

[16] Zhou, Y., Liang, J., Maslov, K. I., and Wang, L. V., "Calibration-free in vivo transverse blood flowmetry based on cross correlation of slow time profiles from photoacoustic microscopy," Opt. Lett., 38(19), 3882-3885 (2013). 
[17] Chen, S.-L., Xie, Z., Carson, P. L., Wang, X., and Guo, L. J., “ In vivo flow speed measurement of capillaries by photoacoustic correlation spectroscopy,” Opt. Lett., 36(20), 4017-4019 (2011).

[18] Liang, J., Zhou, Y., Maslov, K. I., and Wang, L. V., "Cross-correlation-based transverse flow measurements using optical resolution photoacoustic microscopy with a digital micromirror device," BIOMEDO, 18(9), 096004-096004 (2013).

[19] Conkey, D. B., Caravaca-Aguirre, A. M., Dove, J. D., Ju, H., Murray, T. W., and Piestun, R., "Super-resolution photoacoustic imaging through a scattering wall," arXiv preprint arXiv:1310.5736, (2013).

[20] Chaigne, T., Katz, O., Gateau, J., Boccara, C., Gigan, S., and Bossy, E., "Improving photoacoustic-guided focusing in scattering media by spectrally filtered detection," arXiv preprint arXiv:1310.7535, (2013). 